\title{
Late diagnosis of congenital sensorineural hearing impairment: why are detection methods failing?
} Clare Robertson, Shirley Aldridge, Frederick Jarman, Kerryn Saunders, Zeffie Poulakis,
Frank Oberklaid
Centre for Community Child Health and

Ambulatory

Paediatrics, Royal

Children's Hospital,

Flemington Road,

Parkville, Victoria,

Australia 3052

C Robertson

$S$ Aldridge

F Jarman

K Saunders

Z Poulakis

F Oberklaid

Correspondence to:

Dr Aldridge.

Accepted 17 October 1994

\begin{abstract}
This study was designed to look in detail at the paths to diagnosis for a group of 197 children with congenital sensorineural hearing impairment (SNHI), who were diagnosed between 1989 and 1991 in the state of Victoria, Australia. Despite the existence of universal infant screening at 7-9 months by distraction test or questionnaire, the median age at diagnosis for the study group was 18.0 months, with median age at aid fitting of 20.8 months, and median age at commencement of specialised intervention programmes of 22.3 months. Parent questionnaires completed for $143(73 \%)$ of these children showed that $49 \%$ had known risk factors for hearing loss yet only $20 \%$ of them had been referred for audiological assessment before the 7-9 month screen. Only $63 \%$ of those eligible for the 7-9 month screen had received it. Of those children who were screened by distraction test $46 \%$ passed as did $57 \%$ of those screened by questionnaire. Twenty four parents (17\%) described how they had initially 'denied' their own observations of their infants' abnormal hearing behaviour. When concerns were raised with professionals, $10 \%$ of parents were falsely reassured without audiological assessment. Detection methods are failing through a combination of poor screen test efficacy, incomplete population coverage, and parental and professional denial.

(Arch Dis Child 1995; 72: 11-15)
\end{abstract}

Keywords: sensorineural hearing impairment, detection, screening, deafness.

Somewhere between one and two children in every thousand are born each year with a congenital sensorineural hearing impairment (SNHI) of sufficient severity to adversely affect their speech and language development. ${ }^{12}$ Intuitively, early diagnosis, fitting of hearing aids, and entry of these children into specialised intervention programmes would seem to offer the best hope for optimal cognitive, social and developmental outcomes, yet current evidence for this logical benefit is scant. ${ }^{34}$ One possible reason for this is that it is only in recent years that early diagnosis of SNHI has been possible, and the long term outcomes of these children have not been investigated. A large community based study in Europe in the late 1970s found an average age at detection of SNHI of 3 years, ${ }^{5}$ while in the USA the mean age at detection for 1990 was 2.5 years. ${ }^{6}$ Parving in Denmark in 1991 found a median age at detection of 18 months. ${ }^{7}$ While there is a trend toward improvement, the American Joint Committee on Infant Hearing's laudable goal of commencement of habilitation for all hearing impaired children by the age of 6 months $^{8}$ is still far from achieved.

The justification for universal screening for SNHI has always been a subject for debate. ${ }^{9}$ Screening programmes aimed at identification of permanent SNHI will inevitably detect larger numbers of children with transient mild conductive losses, and their passage through the audiological assessment processes may be lengthy and expensive. ${ }^{10}$ Optimal management of such cases remains uncertain, although alerting parents to the possibility of transient hearing difficulties in their child would be regarded by some as beneficial. Neonatal screening is now possible with techniques such as auditory brainstem evoked response, and otoacoustic emissions ${ }^{11} 12$ and universal screening using a combination of these techniques has recently been recommended in the US by the National Institutes of Health (NIH). ${ }^{13}$ There are fears, however, that the costs of such a programme would be overwhelming, its implementation impractical, and its benefits uncertain. ${ }^{9}$ In many parts of the UK and Australia, hearing screening is offered to the entire infant population at 7-9 months using a behavioural technique known as the distraction test. ${ }^{14} 15$ The efficacy of this test has not been well studied in the community setting, and there are mixed reports on its usefulness. ${ }^{1617}$

The objective of this study was to assess the current median age at diagnosis of congenital SNHI in the state of Victoria, Australia, and to examine in detail the paths taken to diagnosis of individual children, with particular reference to the role of the screening programme, and the contribution of professionals to early diagnosis. In Victoria there are approximately 60000 births per year. ${ }^{18}$ During the study period no formal neonatal screening programmes were in operation, although two neonatal units were performing some auditory brainstem evoked response testing on 'at risk' infants, and audiological referral of any infants with risk factors for hearing loss was encouraged. Approximately $50 \%$ of infants with SNHI have an identifiable 'risk factor' for hearing loss and targeted screening of this group has been tried with some success. ${ }^{1920}$ All infants were eligible for 
screening at 7-9 months by distraction testing performed at the local maternal and child health centre by a community nurse. Maternal and child health nurses in Victoria are the principal providers of well child care in the preschool age group. Over $90 \%$ of babies born in the state are seen at least once by one of these nurses. ${ }^{21}$ The distraction test assesses the infant's ability to turn and localise a sound stimulus located outside his/her field of vision. Two testers are needed to perform the test, and in some test centres adequate personnel were not available. In such cases, screening was undertaken by administering a questionnaire which asked parents about their child's hearing ability. Any infants failing an initial screen were retested 4-6 weeks later, a second failure resulting in referral for audiological assessment. All children identified by the audiologists as having a hearing loss sufficient to require a hearing aid were referred to Australian Hearing Services (AHS) for aid fitting, and to an early intervention programme.

\section{Methods}

During the period January 1989-December 1991, 197 Victorian children with congenital SNHI under the age of 5 years were recorded by AHS as having been fitted with hearing aids, and were registered with an early intervention programme.

A parent questionnaire was developed and modified after pilot testing on the parents of 20 children with congenital hearing loss diagnosed outside the study period. During 1992, parents of each of the 197 children were sent this questionnaire with a covering letter, and asked to sign a consent form allowing specified information to be collected from the early intervention programmes and audiological services at which the child had contact. Parents were contacted via the early intervention teams or AHS in the first instance, utilising their last known address on file. This ensured that the study group had no direct access to information on named children, and all data were held in strictest confidence.

The first part of the questionnaire comprised structured questions about the path to diagnosis, while the second part requested parents to write of their experiences in their own words. For responders to the questionnaire, dates were confirmed by comparison with audiological records wherever possible. If no reply was received within six weeks, a reminder letter was sent. In addition, some limited unidentified information was obtained from the audiological and intervention program databases on non-responders to the questionnaire. In the case of the AHS database this consisted of the child's identification number, date of birth, sex, type of hearing loss, initial three frequency average hearing loss, latest three frequency average hearing loss, date of first appointment with AHS, date of fitting of hearing aids, aetiology of hearing loss, and use of English as a second language. For the early intervention teams this included initials, date of birth, sex, date of diagnosis of hearing loss, type of hearing loss, severity of hearing loss, aetiology, date of first and subsequent audiological assessments, type of audiological assessments, and date of enrolment into an early intervention service.

The Daniel's occupational prestige scale was used to grade parents' occupation. ${ }^{22}$ Hearing loss was described as the initial three frequency average $(500,1000$, and $2000 \mathrm{~Hz})$ in the better ear. Hearing loss was then categorised into mild ( $<40 \mathrm{dBHL}$ ), moderate (41-60 dBHL), severe 61-90 dBHL), and profound ( $>90$ $\mathrm{dBHL}$ ). The degree of hearing loss was recorded in each case as the initial three frequency average loss documented on the AHS database. All hearing losses were recorded in $\mathrm{dBHL}$, having been converted from dBA or dBSPL where necessary. The risk factors enquired about were those defined by the Joint Committee on Infant Hearing USA $1982^{23}$ - that is family history of childhood hearing impairment, congenital perinatal infection, anatomical malformations involving the head and neck, birth weight less than 1500 g, hyperbilirubinaemia requiring an exchange transfusion, bacterial meningitis, and severe birth asphyxia.

All information was collected and coded centrally, and entered into an SPSS database. Simple descriptive statistics were generated. Comparison of responders and non-responders with respect to age at diagnosis of SNHI, age at fitting of aids, age at entry to early intervention programmes, and initial three frequency average hearing loss were made using Mann-Whitney $U$ tests. Sex distribution was compared with $\chi^{2}$ test. A stepwise regression analysis was performed to determine the influence of variables 'presence/absence of risk factor', 'father's occupation' (as a measure of socioeconomic score), 'severity of hearing loss', 'screened compared with not screened', 'birth order', 'sex', 'English as a second language status', and 'rural compared with city residence' on mean age at diagnosis of SNHI. This regression was repeated excluding those cases where a risk factor was present. For the parent comments, a constant comparative method of qualitative data analysis was used. ${ }^{24}$ Two of the authors examined all the responses and identified key words or phrases which were grouped together into themes. Comparisons were made between the authors for reliability, and the frequencies of responses were noted.

\section{Results}

Initial quantitative analyses were carried out on all 197 cases in the study group, from information on the AHS and early intervention team databases. Completed parent questionnaires were received from 143 of the 197 cases, giving a $73 \%$ response rate. Responders and non-responders did not differ significantly on age at diagnosis of SNHI, level of hearing loss, sex distribution, use of English as a second language, or place of residence (see table 1). 
Table 1 Comparison of responders and non-responders

\begin{tabular}{llll}
\hline Variable & Responders & Non-responders & p Value \\
\hline Median age at diagnosis (months) & 17 & $18 \cdot 5$ & $0 \cdot 739$ \\
Median initial 3 frequency average loss(dBHL) & 70 & 65 & $0 \cdot 911$ \\
Male:female ratio & $1 \cdot 33: 1$ & $2 \cdot 31: 1$ & $0 \cdot 108$ \\
English as a second language (\%) & $3 \cdot 5$ & $1 \cdot 9$ & $0 \cdot 544$ \\
Ratio of city:rural dwellers & $3 \cdot 47: 1$ & $2: 1$ & $0 \cdot 115$ \\
\hline
\end{tabular}

AGE AT DIAGNOSIS

The overall median age at diagnosis of SNHI for the group was 18.0 months; median age at hearing aid fitting was $20 \cdot 8$ months, and median age at entry to early intervention services was 22.3 months (see table 2 ). Only $30 \%$ of the study group were diagnosed as having a congenital hearing loss before the age of 12 months. Age at diagnosis was related to severity of hearing loss with profound losses being diagnosed significantly earlier than the other degrees of loss (see table 3 ).

Altogether $14.9(75.6 \%)$ of the group had bilateral SNHI, three $(1.5 \%)$ had a congenital conductive loss, $23(11.7 \%)$ had a mixed loss, and one $(0.6 \%)$ had a bilateral high frequency loss only. There were 117 boys and 76 girls in the study group, a ratio of $1 \cdot 53: 1$. Sex was not known for four children.

The remaining analyses were carried out on the responders.

\section{SUSPICION OF HEARING LOSS}

The median age at first suspicion of hearing loss was 9 months. Hearing loss was first suspected by parents in $74(53 \%)$ cases. Maternal and child health nurses were the first to suspect a problem in $22(16 \%)$ cases, other relatives in nine $(6 \%)$, ear, nose, and throat surgeons in four $(3 \%)$, school doctor/nurse in one $(1 \%)$, and others in 30 (21\%).

The initial reasons for suspected hearing loss were stated as lack of response to noise in $81(57.0 \%)$ cases, speech delay in 52 $(36.9 \%)$, behaviour problems in $10(7 \cdot 1 \%)$, recurrent ear infections in $21(14.9 \%)$; known to be at risk in $36(25.5 \%)$, other in 22 $(15 \cdot 6 \%)$, and unknown in one $(0 \cdot 7 \%)$. (More than one reason could be given for initial suspicion.)

Forty nine per cent of the cases had a risk factor for hearing loss, but this was not always the reason for audiological referral. Only 15 of $73(20 \%)$ children with a risk factor were referred to the audiologist before the 7-9 months screen. The median age at diagnosis for those with a risk factor was, however, 12 months compared with 24 months for those without.

Table 2 Age at diagnosis, aid fitting, and entry to early intervention

\begin{tabular}{|c|c|c|c|c|}
\hline & \multirow[b]{2}{*}{ Median } & \multicolumn{2}{|c|}{ Interquartile range } & \multirow[b]{2}{*}{ Mean (SD) } \\
\hline & & 25th Centile & 75th Centile & \\
\hline $\begin{array}{l}\text { Age at diagnosis (months) } \\
\text { Age at fitting of aids (months) } \\
\text { Age at entry to early intervention (months) }\end{array}$ & $\begin{array}{l}18 \cdot 0 \\
20 \cdot 8 \\
22 \cdot 3\end{array}$ & $\begin{array}{l}10 \cdot 5 \\
12 \cdot 9 \\
13 \cdot 7\end{array}$ & $\begin{array}{l}30 \cdot 0 \\
35 \cdot 8 \\
37 \cdot 1\end{array}$ & $\begin{array}{l}21 \cdot 4(13 \cdot 6) \\
25 \cdot 1(14 \cdot 8) \\
26 \cdot 1(15 \cdot 0)\end{array}$ \\
\hline
\end{tabular}

Table 3 Severity of loss and age at diagnosis

\begin{tabular}{lll}
\hline Severity & $\begin{array}{l}\text { No (\%) of } \\
\text { study group }\end{array}$ & $\begin{array}{l}\text { Median (range) age at } \\
\text { diagnosis (months) }\end{array}$ \\
\hline Mild $(<40 \mathrm{~dB})$ & $32(16 \cdot 2)$ & $20 \cdot 5(8-56)$ \\
Moderate $(41-60 \mathrm{~dB})$ & $53(26.9)$ & $20(5-54)$ \\
Severe $(61-90 \mathrm{~dB})$ & $66(33.5)$ & $20(4-54)$ \\
Profound $(>90 \mathrm{~dB})$ & $46(23 \cdot 4)$ & $12(1-24)^{\star}$ \\
\hline
\end{tabular}

${ }^{\star} \mathrm{p}=0.000$ - refers to the age at diagnosis for children with mild/moderate/severe losses compared with those who had profound hearing losses.

\section{SCREENING TESTS}

One hundred and twenty children in the study group were eligible for a screening test at the age of 7-9 months - that is, they had not had a prior hearing test; only $76(63 \%)$ of these infants actually had a screening test performed. Twenty one children were screened by questionnaire and 12 'passed'. Sixty seven were screened by distraction test and 31 passed they included two children with profound and 12 with severe SNHI. Four children actually failed the distraction test screen, but puzzlingly, no further action was taken.

For questionnaire responders only two factors, presence of risk factor $(r=-0 \cdot 32$, $\mathrm{p} \leqslant 0.001)$ and severity of hearing loss $(r=-0.31, \mathrm{p} \leqslant 0.001)$ were significant predictors of age at diagnosis of SNHI, children with risk factors and with more severe forms of SNHI being diagnosed earlier. When only those cases with no risk factors were considered, the only significant predictor variable was severity of hearing loss $(r=-0.45, \mathrm{p} \leqslant 0.001)$.

\section{PARENTS' COMMENTS}

Qualitative data analyses were performed on invited comments from parents. Parents were asked to describe what happened from the time they first suspected that their child had a hearing problem up to the time when aids were fitted; whether they had any suggestions for improving the system of detection and support for children with a hearing loss; and whether they had any other comments. The following principal themes were identified:

\section{Parental and professional denial of the problem} Twenty four parents commented that initially they had 'denied' their observations of abnormal hearing behaviour in their children, hoping they were wrong, and delayed seeking advice. Once advice was sought, however, 14 parents reported that they were falsely reassured by a health professional that there could not be any problem with the hearing.

\section{Complaints about the distraction test}

Fifteen parents openly complained about the screening test with it commonly being described as 'a joke'. The nurses often seemed reluctant to 'fail' the child on the test. One parent wrote: 'I remember how we - my husband and I, the health centre sister and her assistant - tacitly conspired to make sure that our son 'passed' his hearing test. When he didn't react to the rattle we tried and tried 
again until he did ... we went home with evidence in writing that our son's hearing was normal, though, luckily for him, seeds of doubt were germinating inside us'.

\section{Appointment delays}

Nineteen parents commented that they felt the waiting period before initial or follow up appointments with an audiologist was too long.

\section{Inconclusive and incorrect test results}

Twenty three parents reported that they were dissatisfied with the number of inconclusive results obtained by the audiologist. Quite incorrect results from audiology assessments were also mentioned by seven parents. Two parents also reported how doctors carried out their 'own' hearing tests in the surgery and declared the child's hearing normal.

\section{Issues surrounding the insertion of tympanostomy} tubes

Twenty six parents mentioned that their children had had tympanostomy tubes inserted at some point during the diagnostic process, often with the intention of ensuring that middle ear effusions were not contributing to the hearing loss. However, for 18 of these parents it was apparent that they were unaware of the possibility of an underlying SNHI at the time of tube insertion. In two cases tubes were inserted before any form of hearing testing. Two other parents refused tubes which were advised; in both cases the children proved to have pure SNHI.

\section{Discussion}

The late diagnosis of congenital SNHI is a well recognised problem with many possible contributing factors. The data from this study show that despite the existence of an infant screening programme, Victorian children with SNHI are still being diagnosed, fitted with hearing aids, and entered into early intervention programmes much later than desirable. Poor screening test efficacy, suboptimal population coverage, parental and professional denial, and delayed and inconclusive audiology results are all contributing to the problem. Both the 'questionnaire' and the 'distraction test' screen in their present form have an unacceptably high false negative rate.

Although children with risk factors were diagnosed significantly earlier than those without, some children with risk factors were not referred for audiological assessment until the second year of life and beyond. Few children with risk factors were referred for neonatal auditory brainstem evoked response testing, although facilities for this test did exist. These findings are consistent with those of Coplan, who also found a low awareness of the significance of risk factors for hearing loss among the medical profession. ${ }^{25}$ Some doctors seemed unaware that hearing assessment at an early age was possible.
This study confirmed that parents were often the first to suspect the possibility of a hearing loss. ${ }^{26}$ Fifty eight per cent of parents in this study had noted abnormal hearing behaviours in their children compared with $44 \%$ in Watkin's group. ${ }^{27}$ The comments by a number of the parents that they had initially denied their own findings is of real interest as it supports a suspicion by Mindel and Feldman that parents often denied their child's lack of response to sound. ${ }^{28}$ Simmons also suggested that many delays in rehabilitation were actually caused by parents not accepting the diagnosis of hearing impairment. ${ }^{29}$ It is probable that some elements of denial operate throughout all stages of the diagnostic process. Fear of the community's response may be contributing to parents' unwillingness to act on their suspicions. Thus, it is difficult to know whether delayed action by parents is due to lack of knowledge, and hence might be amenable to parental education programmes, or to the more powerful mechanism of subconscious parental denial. It is probably shortsighted to conclude that the answer lies in simply giving parents more information. False reassurance by professionals was a barrier to early diagnosis in this series as in others. ${ }^{3031}$ Again, there is some evidence that there may be denial on the part of the professionals.

While further education of general practitioners, nurses, and paediatricians is needed to ensure that parents' concerns are taken seriously, a close examination of why professionals are so ready to reassure parents in these circumstances is essential. This will involve an exploration of professional attitudes to hearing impairment, and an unwillingness to be 'bearers of bad news'. Even profoundly hearing impaired children may initially babble, and appear outwardly normal. Once parental concern has been expressed it is essential that a formal assessment of the child's hearing status is performed by a trained audiologist. There is evidence that doctors continue to have misplaced confidence in their ability to detect hearing problems. There is a great danger in doctors performing their own hearing tests which are neither valid nor reliable. Desires to allay anxiety may be detrimental when parental fears are well founded.

Anecdotal evidence often suggests that children with SNHI appear to have 'escaped' opportunities for screening. Johnson and Ashurst found that there was an increased risk of hearing impairment among infants who missed their distraction test screen ${ }^{32}$; again this might indicate avoidance by the parents of a test confirming their suspicions. Some children with SNHI are born to similarly affected parents who may not view the condition as a medical problem, ${ }^{33}$ although even this group usually seek early diagnosis. In the study group only $63 \%$ those eligible for screening at 7-9 months were actually tested. Clearly this is suboptimal, but it is not known how these figures compare with those for children with normal hearing. It is of interest that only two factors - presence of risk factor and severity of hearing loss - were significant predictors of age 
at diagnosis. Hence those children who were screened did not appear to have their SNHI detected significantly earlier than those who were not. The lack of effect of screening persisted even when only those children who did not have any risk factors for SNHI were considered, which suggested that the screening programme in place was largely ineffective.

Late diagnosis may arise if the child has a deteriorating loss, for example due to congenital cytomegalovirus infection. This study's analyses excluded children with acquired losses, and no evidence was found of marked deteriorations of hearing status in the children studied: it seems unlikely that deteriorating losses account for the majority of late diagnoses.

The high proportion of children undergoing tympanostomy tube insertion at some point during the diagnostic process was an unexpected finding. Given that there was no indication of this procedure having been performed unless the parents mentioned it in the open ended reply section, it may be that the proportion of children receiving tympanostomy tubes is even greater. It appeared that the operation was usually performed to exclude the possibility that a degree of middle ear effusion or 'glue ear' was contributing to the documented hearing loss. From the parental comments it appeared that, in fact, this was rarely the case. It is readily understandable, however, that both parents and ear, nose, and throat surgeons might wish to proceed with tube insertion if they felt there was any chance that an improvement in hearing ability might result. These findings certainly highlight the limitations of current audiological techniques in distinguishing between a mixed (sensorineural and conductive) hearing loss and a purely conductive loss.

More disturbing, however, is the report by several parents that they were unaware of the possibility of an underlying SNHI before tympanostomy tube insertion, even in cases where the degree of hearing loss at initial assessment made a pure conductive loss very unlikely. Parents were often angry and upset when the true situation was revealed to them, and it is difficult to know whether the problem lies in a true lack of professional understanding of the condition, a miscommunication between parents and professionals, or a kind of 'wishful thinking' or denial on the part of the medical attendants, again hoping not to be the bearers of bad news.

Although screening test efficacy is fundamentally important, the overall success of any screening programme depends on parent and professional knowledge and attitudes, a high take-up rate by the population, and a rapid means of confirmatory testing. Good communication between parents and professionals is essential. Diagnosis and management of SNHI in childhood requires a coordinated, multidisciplinary approach: this is as true for neonatal screening as for screening later in infancy.

These findings have implications for the conduct of other childhood screening programmes aimed at detecting developmental problems where the same psychological defence mechanisms among parents and professionals probably operate. Further studies such as this on the experience of parental consumers would provide invaluable information for the providers of child health services. Such a partnership between parents and professionals is an essential step in the improvement of early detection systems and ultimate long term outcomes.

SA was supported by an NH and MRC PHRDC scholarship. The study was supported in part by a grant from the Victorian Health Promotion Foundation. The authors would like to thank Mr Ian Tyson (AHS), Ms Shirley Dennehy (Taralye), Dr Tim Moore (Monnington), Ms Beth Sutcliffe (Parent Advise Pervice for the Hearing Impaired), and Ms Helen Hill (John questionnaires and collating of data. Special thanks goes to all the parents who participated in the study.

1 Davis A, Wood S. The epidemiology of childhood hearing impairment: factors relevant to planning of services. $\mathrm{Br} \mathscr{F}$ Audiol 1992; 26: 77-90.

2 Upfold L. Isepy J. Childhood deafness in Australia. Incidence and maternal rubella, 1949-1980. Med $\mathcal{F}$ Aust 1982; ii: $323-6$. 3 Markides A. Age at fitting of hearing aids and speech intelligibility. $B r \mathcal{F}$ Audiol 1986; 20: 165-8.

4 Ramkalawan TW, Davis AC. The effects of hearing loss and age at intervention on some language metrics in young hearing at intervention on some language metrics in youn
impaired children. Br f Audiol 1992; 26: 97-107.

5 Martin JAM, Bentzen O, Colley JRT, et al. Childhood deafness in the European Community. Scand Audiol 1981; 10: 165-74.

6 Gustason G. Early identification of hearing impaired infants: a review of Israeli and American progress. Volta Review 1989; 91: 291-5.

7 Parving A. Detection of the infant with congenital/early acquired hearing disability. Acta Otolaryngol (Stockh) 1991; 482 (suppl) $111-6$

8 Joint Committee on Infant Hearing 1990 position statement American Speech-Language Association Supplement 1991 (Mar); 33(5): 3-6.

9 Bess FH, Paradise JL. Universal screening for infant hearing impairment: not simple, not risk free, not necessarily beneficial, and not presently justified. Pediatrics 1994; 93 $330-4$

10 Mott A, Emond A. What is the role of the distraction test of hearing? Arch Dis Child 1994; 70: 10-3.

11 Haggard MP. Hearing screening in children - state of the art(s) Arch Dis Child 1990; 65: 1193-5.

12 Stevens JC, Webb HD, Hutchinson J, Connell J, Smith MF Buffin JT. Click evoked otoacoustic emissions compared with brainstem electric response. Arch Dis Child 1989; 64: 1105-11

NIH Consensus Statement. Early identification of hearing impairment in infants and young children. Bethesda, $M D$ Office of Medical Applications of Research, NIH, March 1-3,
1993; 11: 1-24. 4 1993; 11: 1-24,

Stewart Brown S, Haslum MN. Screening for hearing loss in childhood: a study of national practice. $B M F$ 1987; 294:

Ewing IR, Ewing AWG. The ascertainment of deafness in infancy and early childhood. F Laryngol Otol 1944; 59: 309-33. Scanlon PE, Bamford JM. Early identification of hearing loss:
screening and surveillance methods. Arch Dis Child 1990; 65: 479-85.

17 McCormick B. Hearing screening by health visitors: a critical appraisal of the distraction test. Health Visitor 1983; 56: 449-51.

18 Lumley J. Births in Victoria: a statistical overview. Having a baby in Victoria: final report of the ministerial review of birthing services in Victoria. Victoria: Health Department, 1990: 10.

19 Mauk GW, White KR, Motensen LB, Behrens TR. The effectiveness of screening programs base $A$ on high-risk characteris1991; 12: 312-9.

20 Diefendorf A, Reitz PS, Cox JB. The joint committee on infant hearing 1990 position statement: a closer look. Infants and Young Children 1992; 5: v-xi.

21 Ochiltree G. An ear to listen to and a shoulder to cry on. Family Matters Nov 1990; No 27: 44-8.

22 Daniel A. Power, privilege and prestige: occupations in Australia Melbourne: Longman Cheshire Pty Ltd, 1983: 196-206.

23 Joint Committee on Infant Hearing. Position statement. Pediatrics 1982; 80: 496-7.

24 Miles MB, Huberman AM. Qualitative data analysis. Beverley Hills, CA: Sage, 1984

5 Coplan J. Deafness: ever heard of it? delayed recognition of permanent hearing loss. Pediatrics 1987; 79: 206-13.

26 Macfarlane A. Child health services in the community: making them work. BMF 1986; 293: 222-3.

27 Watkin PM, Baldwin M, Laoide S. Parental suspicion and identification of hearing impairment. Arch Dis Child 1990; 65: 846-50.

28 Mindel ED, Feldman V. The impact of deaf children on their families. In: Mindel ED, Vernon M, eds. They grow in silence. understanding deaf children and adults. 2nd Ed. Boston: CollegeHill, 1987: 1-30.

29 Simmons FB. Diagnosis and rehabilitation of deaf newborns. American Speech-Language Association 1980; 22: 475.

ing loss: listen to parents. Clin Pediatr (Phila) of hearing los:

31 Upfold LJ. The early detection of childhood deafness. Med 7 Aust 1978; ii: $507-10$.

32 Johnson A, Ashurst $\mathrm{H}$ for the Steering Committee, Oxford Region Child Development Project. Screening for 65: 841-5.

33 Mohay H. Deafness in children. Med F Aust 1991; 154: 372-4. 